ISSN 0103-8478

\title{
Sistema setorial do biodiesel no Rio Grande do Sul: caracterização e oportunidades para a consolidação de um sistema inovativo em agroenergia
}

\author{
Biodiesel sector system in Rio Grande do Sul: characterization and opportunities for consolidating \\ an innovative system for bioenergy
}

\author{
Mateus Sangoi Frozza ${ }^{\mathrm{I}}$ Ana Lúcia Tatsch ${ }^{\mathrm{II}}$
}

\section{RESUMO}

Este trabalho adota o enfoque neo-schumpeteriano de sistemas de inovação para caracterizar o Sistema Setorial do Biodiesel no Rio Grande do Sul. À luz desse enfoque, examinaramse as possíveis interações que se estabelecem entre as empresas industriais produtoras de biodiesel no estado e outros agentes, como universidades e institutos de pesquisa. O estudo foi levado a cabo mediante a sistematização de um conjunto de dados secundários de diferentes fontes, além da obtenção de dados primários, a partir de entrevistas orientadas por questionário estruturado. Constatou-se que as firmas inovam de forma restrita. Implementam inovações tecnológicas exclusivamente de processo e realizam mudanças organizacionais. Verificou-se ainda que o aparato institucional de pesquisa e ensino presente no estado, embora gerador de conhecimentos importantes, interage pouco com o setor produtivo privado. De toda forma, percebem-se oportunidades para o Rio Grande do Sul tornar-se referência na produção do biodiesel no País.

Palavras-chave: sistemas inovativos, biodiesel, Rio Grande do Sul.

\section{ABSTRACT}

This paper adopts the approach of neo-schumpeterian innovation systems to characterize the Sectorial System of Biodiesel in Rio Grande do Sul, Brazil. Based on this approach, it was examined the possible interactions that take place between biodiesel companies in the state and other agents such as universities and research institutes. The study was conducted by the systematization of a secondary set data from different sources. In addition, to obtainprimary data, interviews were conducted guided by a structured questionnaire. It was found that firms innovate narrowly, implementing technological innovations exclusively in process and realizing organizational changes. It was also found that the institutional apparatus research and educational in this state, while generating important knowledge, interacts little with the private productive sector. Anyway, it was found opportunities to Rio Grande do Sul become a reference in the production of biodiesel in Brazil.

Key words: innovative systems, Biodiesel, Rio Grande do Sul.

\section{INTRODUÇÃO}

Em anos recentes, várias ações ligadas a novas fontes de energia tem se desenvolvido no Brasil. Os primeiros esforços significativos estiveram relacionados à constituição do Programa Nacional do Álcool (PROÁLCOOL) na década de 70. Mais recentemente, em 2005, com o Plano Nacional de Agroenergia e, em 2007, com o Programa Nacional de Produção e Uso do Biodiesel (PNPB), desenham-se as principais políticas voltadas para os biocombustíveis no País. Segundo estudo do IPEA, a participação de instituições estatais em conjunto com redes de pesquisa e atores sociais, como representações de classes e sindicatos rurais, constitui-se em um diferencial brasileiro na promoção do biodiesel (PÊGO \& CAMPOS NETO, 2010). Nesse cenário, também foi instituído o Programa Gaúcho de Biodiesel (PROBIODIESEL/RS), já que a produção e a comercialização do biodiesel se configura em uma oportunidade de desenvolvimento econômico para o Rio Grande do Sul (RS).

Diante dessas perspectivas, justificase um olhar sobre a realidade gaúcha no que tange

'Centro Universitário Franciscano (UNIFRA), 97010-491, Santa Maria, RS, Brasil. E-mail: mateusfrozza@gmail.com. Autor para correspondência.

IDepartamento de Economia e Relações Internacionais, Universidade Federal do Rio Grande do Sul (UFRGS), Porto Alegre, RS, Brasil. Recebido 29.05.13 Aprovado 31.03.14 Devolvido pelo autor 07.08.14 CR-2013-0756.R2 
à produção do biodiesel. Para tanto, adota-se como referencial teórico para a análise o conceito de sistema inovativo. Tal conceito proposto pelos evolucionistas e neo-schumpeterianos permite um olhar sistêmico sobre os atores e as relações que se estabelecem entre eles, especialmente no que tange aos processos de geração, uso e difusão de conhecimentos e inovação. A concepção de Sistemas de Inovação permite uma análise do processo inovativo em diferentes âmbitos (nacional, regional e setorial). Trata-se de uma construção teórica alicerçada nos estudos de FREEMAN (1995), LUNDVALL (1992), NELSON (1993), PATEL \& PAVITT (1994), BRESCHI \& MALERBA (1997), MALERBA (2002), e COOKE (2001; 2009).

O sistema de inovação compreende um arranjo institucional que envolve uma série de participantes (empresas e suas redes de cooperação, governos, universidades e institutos de pesquisa, setor financeiro, entre outros) que interagem e articulamse entre si. Nesse contexto, a inovação é vista como um processo social e sistêmico, resultado não só das capacidades inovativas das firmas individuais, mas da interação dessas com as demais organizações que fazem parte do sistema. O comportamento desses agentes é também influenciado pelo contexto institucional (EDQUIST, 1997).

Outras dimensões do sistema de inovação, além da nacional, também podem ser exploradas. Dada determinadas especificidades quanto ao espaço geográfico, à matriz institucional, às características tecnológicas e de fabricação, diferentes recortes de análise podem ser realizados, como o regional (COOKE, 2001; 2009) e o setorial (BRESCHI \& MALERBA, 1997; MALERBA, 2002). Essas análises também podem ser complementares, como a realizada neste trabalho.

Este artigo examina justamente a configuração de um sistema particular, definido em âmbito regional (Rio Grande do Sul) e setorial (Biodiesel), isto é, o Sistema Inovativo do Biodiesel no RS. Visa identificar os agentes e atores envolvidos nesse sistema, bem como examinar possíveis interações e cooperação entre eles, objetivando a troca de informações científicas e tecnológicas. A contribuição do estudo está na análise desse sistema que, embora relevante para o País, foi ainda pouco estudado. Suas conclusões podem orientar as ações de política, visando a melhor explorar as oportunidades que se apresentam ao RS e ao Brasil no que tange aos bioscombustíveis.

O texto está estruturado em três seções, além desta introdução. Na primeira, descrevem-se os procedimentos metodológicos. Na segunda seção, são discutidos os resultados. Por fim, na terceira, apresentam-se as considerações finais.

\section{METODOLOGIA}

O estudo teve caráter teórico-empírico. Foi realizado através de levantamento bibliográfico e de pesquisa de dados secundários e primários. $\mathrm{O}$ ponto de partida foi a coleta e análise de informações secundárias. Isso se traduziu em um esforço de caracterização do conjunto de organizações envolvidas na produção, na geração e difusão de conhecimentos, na capacitação, representação, fomento e políticas públicas voltadas ao biodiesel do RS. A partir daí, selecionaram-se os agentes a serem investigados empiricamente. A pesquisa de campo, realizada em 2010, ocorreu junto às empresas produtoras de biodiesel localizadas no estado ${ }^{\mathbf{a}}$, bem como junto a diferentes organizações, como institutos de pesquisa e universidades. $\mathrm{O}$ instrumental empregado nessa etapa consistiu de um questionário estruturado e de roteiros de entrevistas, ambos aplicados presencialmente. O questionário $^{\mathbf{b}}$ foi direcionado aos agentes produtivos - empresas industriais -, enquanto que os roteiros de entrevistas abordaram questões específicas, relacionadas à atuação e envolvimento das demais instituições. O questionário foi estruturado a partir de cinco blocos de perguntas: I- Identificação da empresa; II- Produção, mercados e emprego; IIIInovação, cooperação e aprendizado; IV- Estrutura, governança e vantagens associadas ao ambiente local; e V-Políticas públicas e formas de financiamento, em que foram distribuídas 48 perguntas.

As empresas que participaram do estudo foram: a Brasil Ecodiesel, a Granol, a OLEOPLAN e a BSBIOS. Todas, juntas, quando operam com capacidade total, podem produzir um volume de aproximadamente 450 milhões de litros, o que representa elevado percentual da produção nacional.

Participaram também da pesquisa empírica a Universidade Federal do Rio Grande do Sul (UFRGS), a Universidade do Vale do Rio dos Sinos (UNISINOS), a Fundação Estadual de Proteção Ambiental Henrique Luís Roessler (FEPAM), a Fundação de Ciência e Tecnologia (CIENTEC) e a Fundação Estadual de Pesquisa Agropecuária (FEPAGRO). Essas instituições fizeram parte do Projeto Estruturante de Agroenergia do Estado do RS, que será apresentado adiante. Os entrevistados em cada uma dessas instituições foram os coordenadores dos subprojetos vinculados a esse Projeto Estruturante. Também a Caixa Estadual 
S.A. - Agência de Fomento/RS (CaixaRS), hoje BADESUL Desenvolvimento - Agência de Fomento/ RS, na figura de seu diretor, e a União Brasileira do Biodiesel (UBRABIO), por meio do seu presidente executivo, foram alvo da investigação direta.

Cuidou-se para que os respondentes tivessem cargos de nível alto ou médio, de modo que detivessem as informações solicitadas. Assim, no caso das empresas, foram entrevistados, especialmente, profissionais ligados às diretorias de manufatura/ produto, fomento, comercialização e produção, sendo um representante de cada empresa. Esses, somados aos respondentes das demais instituições participantes do estudo, perfazem um total de onze entrevistados.

As informações coletadas na investigação direta foram tratadas tanto a partir da análise de conteúdo quanto por sistematização quantitativa, calcada sobretudo em estatística descritiva.

\section{RESULTADOS E DISCUSSÃO}

O enfoque sistêmico buscado nesta análise implica a construção de um esquema analítico, cujo foco são os seus atores, assim como os fluxos de conhecimento e as formas de interação, cooperação e aprendizado que se estabelecem entre eles.

No que tange ao arranjo institucional voltado ao biodiesel no estado, são vários os organismos estatais atuantes. A secretaria de Ciência, Inovação e Desenvolvimento Tecnológico (SCIT) é que coordena o Programa Gaúcho de Biodiesel (PROBIODIESEL/ RS), instituído em 2003 em consonância com o PNPB. A coordenação executiva cabe à Fundação de Ciência e Tecnologia (CIENTEC), mas várias outras secretarias têm ações voltadas ao Programa.

No âmbito desse Programa e visando a sua consolidação, foi realizado o Projeto Estruturante de Agroenergia do Estado do RS no período de 2008 a 2011, o qual mobilizou várias instituições no estado. O Projeto contou com recursos da Financiadora de Estudos e Projetos (FINEP) e da Fundação de Amparo à Pesquisa do Estado do Rio Grande do Sul (FAPERGS), totalizando um montante de R\$ 4,166 milhões. As instituições co-executoras líderes foram a UFRGS, a FEPAM, a FEPAGRO, a CIENTEC e a UNISINOS. Além dessas instituições, o projeto envolveu a Empresa Brasileira de Pesquisa Agropecuária (EMBRAPA), a Empresa de Assistência Técnica e Extensão Rural (EMATER), cooperativas e várias universidades no estado, bem como as empresas industriais produtoras de biodiesel. Dentre os objetivos do Projeto, estavam o desenvolvimento de tecnologias para o aproveitamento de coprodutos, a caracterização e controle de qualidade dos insumosprodutos, e a qualificação de recursos humanos. Além dissso, visou a disponibilizar um banco de dados dos parâmetros produtivos (agronômicos e industriais), socioeconômicos, biotecnológicos e ambientais, bem como ofertar uma base de competências para prestação de serviços de apoio à agroenergia. Segundo avaliação dos coordenadores dos projetos das co-executoras líderes, parte das metas propostas pelo Projeto foram cumpridas e resultados foram gerados, como: o estabelecimento de protocolos de controle de qualidade; o zoneamento agroclimático para as culturas da mamona, canola e mandioca; a geração de informações técnicas para secagem, armazenamento e conservação dos insumos-produtos, que se apresentam como etapas de gargalo; e a análise de emissões veiculares, utilizando diferentes misturas de biodiesel ao diesel convencional. Em contrapartida, os coordenadores dos subprojetos assinalaram a dificuldade de estabelecer uma interação maior entre as diferentes equipes envolvidas no Projeto Estruturante, de modo a promover troca de informações entre os grupos de pesquisadores.

Ainda com a meta de fomentar a produção do biodiesel no RS, o governo, sob a coordenação da Secretaria da Agricultura, Pecuária e Agronegócio (SEAPA/RS), constituiu a Câmara Temática de Agroenergia em 2011. O intuito foi unir os diferentes atores envolvidos com a produção de biocombustíveis (biodiesel, etanol, biogás) e discutir questões pertinentes à agroenergia.

Com relação aos investimentos e financiamentos, o governo do Estado, através do Fundo de Operação Empresa do Estado do Rio Grande do Sul (FUNDOPEM/RS) e do Programa de Harmonização do Desenvolvimento Industrial do Rio Grande do Sul (INTEGRAR/RS), contemplou as empresas de biodiesel no estado com benefícios financeiros previstos em lei.

Os investimentos oriundos da parceria entre CaixaRS, Banco Regional de Desenvolvimento do Extremo Sul (BRDE) e Banco Nacional de Desenvolvimento Econômico e Social (BNDES) proporcionaram as primeiras iniciativas governamentais que impulsionaram a industrialização, bem como a comercialização do biodiesel no RS. A Agência de Fomento do Estado, anteriormente CaixaRS e atualmente Badesul, pelo fato de ser uma importante intermediária na concessão de crédito (uma vez que é o BNDES o principal agente financiador de projetos para o biodiesel no País), exerce um papel fundamental para o fomento e a consolidação do biodiesel no estado. 
Com relação às empresas industriais produtoras de biodiesel, importantes atores no sistema ora em análise, as quatro que atuavam à época da pesquisa foram visitadas e entrevistadas. Todas são associadas a UBRABIO.

A primeira empresa a trabalhar com Biodiesel no RS foi a Óleos Vegetais Planalto (OLEOPLAN S.A), cuja planta industrial situa-se em Veranópolis. Com capital inteiramente nacional, dedica-se à extração de óleos vegetais desde 1980. Em 2007, ingressou no segmento de biodiesel. A tecnologia que emprega é oriunda de firmas italianas, francesas e alemãs. Sua planta industrial foi idealizada em conjunto com o Instituto Alberto Luiz Coimbra de Pós-graduação e Pesquisa em Engenharia (COPPE) da Universidade Federal do Rio de Janeiro (UFRJ), que é também responsável pela sua manutenção. O principal produto da empresa é o biodiesel, que representa $50 \%$ da produção total; o restante é destinado à produção de farelo $(40 \%)$ e de glicerina $(10 \%)$.

A Brasil Ecodiesel, localizada em Rosário do Sul, foi a segunda empresa a operar no estado a partir de $2005^{\mathrm{c}}$. Além dessa planta gaúcha, a empresa possui outras quatro no Nordeste e uma no Norte do País. O capital controlador da empresa constituise de ações negociadas na Bolsa de Valores. A planta gaúcha foi instalada pela empresa TECBIO, resultado da parceria entre a Universidade Federal do Ceará (UFCE) e o professor Expedito José Parente, pioneiro na produção de biodiesel no Brasil. A TECBIO é responsável pela manutenção da planta. $\mathrm{O}$ biodiesel corresponde a $95 \%$ de sua produção. Outro subproduto da unidade é a glicerina, que corresponde a $5 \%$ do total produzido.

A BSBIOS, fundada em 2005 e operando em Passo Fundo a partir de 2007, é a terceira firma a funcionar no RS com a finalidade específica de produção de biodiesel. A empresa tem capital

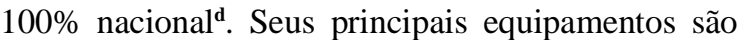
de tecnologia americana, da Crown Iron Works Company, cuja subsidiária brasileira - Intecnial S.A. - localiza-se em Erechim, no RS. O Biodiesel responde por $70 \%$ de sua produção, acompanhado de $25 \%$ de farelo e $5 \%$ de glicerina. A empresa tem como estratégia não esmagar os grãos adquiridos junto aos agricultores, mas sim recorrer às esmagadoras da região que disponibilizam este serviço. A empresa também compra o óleo vegetal disponível no mercado local e regional.

Segundo o coordenador de fomento da BSBIOS, a escolha por Passo Fundo se deveu à sua localização, já que faz parte de um pólo produtivo de oleaginosas e está próxima de cooperativas, produtores e esmagadoras regionais de oleaginosas. Outro aspecto que influenciou na localização da empresa foi o aparato tecnológico ali disponível. Isso se dá pela presença da Universidade de Passo Fundo (UPF), da EMBRAPA e da EMATER.

A quarta empresa a operar no RS foi a Granol Indústria, Comércio e Exportação. A empresa paulista iniciou o projeto no município de Cachoeira do Sul, em 2005. Reativou a planta da extinta Central de Recebimento de Grãos do Sul (Centralsul), pertencente ao Governo Federal. O local ficou desativado por mais de 20 anos, mas sua estrutura foi preservada. A unidade da Granol no RS se vale de tecnologia do grupo alemão GEA. A produção corresponde a $8 \%$ de farelo, $2 \%$ de glicerina e $90 \%$ de biodiesel.

A principal matéria-prima utilizada pelas quatro firmas é a soja; comprada em sua grande maioria no noroeste do estado. A Granol e a BSBIOS usam ainda soja proveniente do Mato Grosso do Sul. A OLEOPLAN e a BSBIOS utilizam também canola como insumo, adquirida igualmente no noroeste gaúcho. Todos os fabricantes temem por possíveis crises de abastecimento de matéria-prima, não só devido à expansão da produção de biodiesel no País, mas especialmente em função da ampliação da porcentagem da mistura autorizada pela ANPe . A dependência da soja aporta também risco, em função dessa commodity ser cotada no mercado internacional e ser sensível às oscilações externas.

As sobras do processo de obtenção do biodiesel, como a glicerina e o farelo, são comercializadas pelas empresas. Na OLEOPLAN, a glicerina é exportada para países da Ásia e Oceania. A Brasil Ecodiesel vende esse produto para a indústria de cosméticos e de siderurgia. A glicerina da empresa BSBIOS é vendida para a indústria de sabão e da Granol é destinada à exportação. Seu farelo é destinado para a alimentação animal, principalmente, para a indústria avícola. Os pesquisados ressaltam que falta demanda por glicerina no mercado, o que acarreta um custo extra de armazenamento desse produto.

A comercialização do biodiesel, no País, dá-se para um único cliente, a Petrobras. A empresa é responsável pela regulamentação do setor, através de leilões realizados trimestralmente na ANP. Na opinião dos entrevistados, o grande entrave dos leilões é que estes não diferenciam a qualidade do produto entregue, mas sim a quantidade estipulada pela ANP. Para as empresas, esse tipo de prática limita a comercialização e a produção do produto no Brasil. 
Com relação à inovação, as empresas pesquisadasassinalaramquerealizamsomentealgumas inovações de processo, as quais compreendem, por exemplo, a compra de equipamentos mais modernos e adaptações industriais, visando particularmente redução de custos de produção. Tais inovações permitiram, segundo os informantes, reduzir o consumo de matérias-primas, de energia e o impacto no meio ambiente, e ainda elevar a produtividade. A Granol e a BSBIOS destacam que suas capacidades de inovar em processo acabam se restringindo, em função dos seus equipamentos serem importados e, portanto, dependerem de tecnologia e know-how estrangeiros.

Dada a tradição da indústria gaúcha no segmento metal-mecânico e na produção de bens de capital, tem-se aqui uma oportunidade de desenvolver a fabricação de equipamentos nacionais voltados à produção de biocombustíveis. A reconhecida qualificação técnica da pesquisa realizada na área das engenharias no estado pode auxiliar nessa direção. Em consequência, a cadeia produtiva do biodiesel pode ser adensada.

Inovações organizacionais também ocorrem. A Brasil Ecodiesel, por exemplo, relata que tais inovações decorrem muitas vezes da implementação de novos métodos de gerenciamento para atender normas de certificações.

Segundo as empresas, elas próprias são as principais responsáveis pelo desenvolvimento da inovação. Nesse sentido, valem-se de fontes de informação para a inovação, sobretudo internas à empresa, ligadas especialmente à área de produção. Entre as empresas pesquisadas, somente a Granol possui laboratório voltado à pesquisa e desenvolvimento, no qual realiza testes de qualidade e aperfeiçoa seu produto. A Brasil Ecodiesel possui laboratório de P\&D somente na matriz no Ceará.

Com relação às fontes externas voltadas para a inovação, a OLEOPLAN sublinha a troca de informações que ocorre com a COPPE/UFRJ para ajustes no processo fabril. A BSBIOS cita os fornecedores de insumos e matéria-prima como importantes fontes de informação externa para o aprendizado. Segundo essa empresa, a parceria com fornecedores de sementes estimula a produção de matrizes produtivas alternativas (canola e girassol). Menciona ainda as interações que estabelece com a EMBRAPA, a EMATER, e com a UPF para a realização de experimentos técnicos e desenvolvimento de matérias-primas alternativas. Com a EMATER, possui convênio assinado na área de cooperação técnica. O órgão estadual atua de forma conjunta com a BSBIOS na busca de produtores em potencial para cultivar outras oleaginosas que não a soja. Na UPF, encontra-se instalada a incubadora de produção de biodiesel que visa a propor soluções e qualificar mão de obra na região sul do estado.

Buscando qualificar e treinar mão de obra, dada a carência percebida pelo conjunto das firmas entrevistadas, a Brasil Ecodiesel estabeleceu parceria com a Universidade da Região da Campanha (URCAMP), Campus São Gabriel. O objetivo foi criar curso de graduação ou técnico na área de agronegócio e geração de energia. Buscou ainda parceria com o Serviço Nacional de Aprendizagem Comercial (Senac) para propor cursos de assistência técnica e manutenção. Já a OLEOPLAN procurou apoio no campus Cachoeira do Sul do Instituto Federal do Rio Grande do Sul (IFERS) para suprir a carência de mão de obra e fomenter a pesquisa voltada ao biodiesel.

Outras parcerias também são estabelecidas, especialmente com os produtores rurais. A OLEOPLAN relaciona-se com produtores rurais espalhados em mais de duzentos municípios gaúchos. A BSBIOS possui vínculos diretos de plantio com agricultores da sua região, disponibilizando sementes e suporte técnico. Tal assistência técnica ao produtor ocorre em cooperação com a EMATER. Mantém também parceria com cooperativas da região, como a Cooperativa Mista de São Luiz Gonzaga (COOPERMIL) e a Cooperativa Agropecuária e Industrial (COTRIJUÍ) em Ijuí. As cooperativas agrícolas, as cerealistas e as associações de produtores rurais são importantes parceiras comerciais da empresa. Para mobilizar os agricultores familiares, a BSBIOS conta com o apoio da Federação dos Trabalhadores na Agricultura no RS (FETAG/RS), que atua na intermediação entre os agricultores e a empresa. A aproximação das empresas investigadas com os produtores rurais é estimulada pelos critérios de concessão do Selo Socialf .

Entre as próprias empresas produtores de biodiesel no estado, não se verificou nenhuma cooperação, mesmo que informal. $\mathrm{O}$ diretor de operações da Brasil Ecodiesel mencionou que fez uma tentativa de criar um fórum de gerentes, com representantes de outras empresas do setor, com o intuito de discutir temas relacionados ao meio ambiente, ao aproveitamento de resíduos e às dificuldades de acesso às fontes de financiamento, mas a iniciativa não teve sucesso. Especula que não há interesse na troca de experiências e de informações entre as empresas. Atribui esse fato à ausência local da cultura de cooperação e à falta de confiança mútua entre as empresas. Outro informante ressalta que as 
empresas não cooperam nos aspectos financeiros e ambientais devido às incertezas no debate sobre o marco regulatório da mistura do biodiesel ao diesel. De todo modo, embora concorrentes, há oportunidade das empresas cooperarem em áreas em que todas podem se beneficiar.

\section{CONCLUSÃO}

A partir do exame das fontes secundárias consultadas e da pesquisa de campo realizada, podese concluir que o RS possui uma infraestrutura científica e tecnológica com capacidade de pesquisa e geração de conhecimentos no campo dos biocombustíveis. No entanto, embora se identifique a existência de instituições de pesquisa e ensino, percebeu-se ainda uma tênue interação dessas com o setor produtivo privado. No geral, verificou-se uma baixa interação das empresas com as universidades e institutos de pesquisa. Os pontos de interação das firmas investigadas com as universidade calcam-se sobretudo na capacitação e no treinamento da mão de obra e não em pesquisa científica e transferência de tecnologia. A indústria perde assim capacidade de se valer de estudos científicos para avançar na diversificação de matérias-primas e reduzir a dependência da soja.

Além disso, constatou-se que as firmas inovam de forma restrita. Quando o fazem, inovam tecnologicamente exclusivamente em processo, via compra de maquinário e ajustes no processo de fabricação. Algumas firmas implementam também mudanças organizacionais. Percebem-se como as principais responsáveis por tais inovações e apontam a própria experiência no processo de produção como fonte importante de aprendizado. As empresas identificam ainda os produtores rurais como importantes parceiros, com os quais estabelecem normalmente contratos de compra e venda, o que é estimulado pelos requisitos para a concessão do Selo Social.

A partir do diagnóstico realizado, constatase que o sistema setorial inovativo do biodiesel gaúcho não está, de forma nenhuma, consolidado. Isso porque a articulação institucional entre os sistemas científicotecnológicos, financeiro, educacional e produtivo alcançam ainda um baixo grau de amadurecimento. Tais conclusões corroboram outras investigações sobre sistemas inovativos no Brasilg. Somente a partir de diagnósticos dessa natureza pode-se descortinar nossa realidade, bem como traçar estratégias para superar os gargalos e dinamizar as potencialidades.

No RS, há importantes oportunidades para o desenvolvimento e consolidação desse sistema setorial. Dada a estrutura fundiária do estado, o papel das cooperativas e da agricultura familiar, bem como o fácil acesso às fontes de matéria-prima e a presença de um aparato institucional de ensino e pesquisa com condições de gerar conhecimentos científicostecnológicos, o RS tem condições de tornar-se referência na produção de biodiesel no País.

Para tanto, é importante avançar nas políticas e ações de fomento, na ampliação de linhas de financiamento, no apoio à pesquisa e inovação tecnológica, especialmente para criar alternativas de matérias-primas e melhorar o aproveitamento dos coprodutos gerados, assim como no fortalecimento das articulações entre o sistema produtivo privado e as instituições de apoio à ciência e tecnologia.

\section{FONTE DE AQUISIÇÃO}

a - Atualmente, encontram-se instaladas no RS seis empresas além das quatro pesquisadas. Tais empresas não fizeram parte do presente estudo por que, à época, ou estavam em fase de instalação ou em processo de regulamentação junto à Agência Nacional do Petróleo, Gás Natural e Biocombustíveis (ANP).

b - Tal instrumento foi elaborado levando em conta o questionário formulado pela Pesquisa de Inovação Tecnológica (Pintec) do IBGE.

c - Em 2011, a empresa gaúcha Camera compra essa usina da Brasil Ecodiesel.

d - Em 2011, 50\% da companhia foi vendida para a Petrobras Biocombustível (PBio), subsidiária da estatal brasileira de petróleo. Com a associação, a BSBIOS se transformou na maior produtora de biodiesel no RS.

e - Conforme a legislação brasileira, a adição obrigatória do biodiesel no diesel fóssil segue os seguintes parâmetros: $2 \%$ de 2005 a 2007, de 3\% de 2008 a 2012, e de 5\% a partir de 2013. Porém, em 2009, já se alcançou $4 \%$ e, em 2010, 5\%. Essa mistura de $5 \%$ de biodiesel e $95 \%$ de diesel é denominada de B5.

f - O "Selo Combustível Social" é atribuído pelo Ministério do Desenvolvimento Agrário às empresas produtoras de biodieesel que cumprem uma série de critérios, como estabelecer contratos de compra e venda de matéria-prima com agricultores familiares ou com suas cooperativas e prestar capacitação e assitência técnica a eles. Uma vez certificado, o produtor tem acesso a alíquotas de PIS/Pasep e Cofins diferenciadas. Com essa ação, o governo federal visa a buscar apoio à agricultura familiar e promover a inserção social.

g - Também seguindo a tipologia de PATEL E PAVITT (1994), que classificam os sistemas nacionais de inovação em maduros, intermediários e incompletos, ALBUQUERQUE (1996), VIOTTI (2003) e SUZIGAN e ALBUQUERQUE (2011) atestam que o sistema nacional de inovação brasileiro está situado em um nível intermediário de construção. Afirmam que o Brasil possui um sistema mais caracterizado como de ciência e tecnologia do que como sistema de inovação propriamente dito. Por isso, VIOTTI (2003) propõe dois tipos de sistemas nacionais de mudança técnica: os liderados pelo processo de inovação, característico dos países desenvolvidos, e aquele dominado pelo aprendizado tecnológico, característico das economias em desenvolvimento. O aprendizado tecnológico abrange a absorção de inovações geradas em outras economias e a adaptação e aperfeiçoamento dessas. 


\section{COMITÊ DE ÉTICA E BIOSSEGURANÇA}

Declaração dos autores assumindo a responsabilidade por não possuir aprovação do Comitê de Ética: Declaro como minha interira responsabilidade os dados e as entrevistas apresentados no artigo intitulado de "O Sistema inovativo de biodisel Inovação cooperação e aprendizagem nas empresas localizadas no Rio Grande do Sul".

\section{REFERÊNCIAS}

ALBUQUERQUE, E.M. Sistema nacional de inovação no brasil: uma análise introdutória a partir de dados disponíveis sobre a ciência e a tecnologia. Revista de Economia Política, v.16, n.3, p.56-72, 1996. Disponível em: <http://www.rep.org.br/PDF/63-6. PDF>. Acesso em: 09 mar. 2010.

BRESCHI, S.; MALERBA, F. Sectoral innovation systems: technological regimes, schumpeterian dynamics, and spatial boundaries. In: EDQUIST, C. (Ed.). Systems of Innovation: technologies, institutions and organizations. London: Pinter, 1997. p.130-156.

COOKE, P. Regional innovation system, asymmetric knowledge and the legacies of learning. In: RUTTEN, R.; BOEKEMA, F. (Eds.). The learning region: foudations, state of the art, future. Disponível em: <www.business.aau.dk/ike/upcoming/cooke.pdf>. Acesso em: 22 jun. 2009.

Regional innovation systems, clusters and the knowledge economy. Industrial and Corporate Change, v.10, n.4, p.945974, 2001. Disponível em: <http://icc.oxfordjournals.org/ content/10/4/945.short>. Acesso em: 10 mar. 2010. doi: 10.1093/ icc/10.4.945

EDQUIST, C. (Ed.). Systems of innovation: technologies, institutions and organizations. London: Pinter, 1997. Total de p. 432 .

FREEMAN, C. The 'national system of innovation' in historical perspective. Cambridge Journal of Economics, v.19, p.5-
24, 1995. Disponível em: <http://cje.oxfordjournals.org/ content/19/1/5.full.pdf+html >. Acesso em: 09 mar. 2010.

LUNDVALL, B-A. (Ed). National systems of innovation: towards a theory of innovation and interactive leaning. London: Printer Publishers, 1992. Total de p. 388.

MALERBA, F. Sectoral system of innovation and production. Research Policy, v.31, p.247-264, 2002. Disponível em: <http:// www.sciencedirect.com/science/article/pii/S0048733301001391>. Acesso em: 15 mar. 2010. doi: 10.1016/S0048-7333(01)00139-1

NELSON, R. (Ed.). National innovation systems: a comparative analysis. Oxford: Oxford University Press, 1993. Total de p.541.

PATEL, P.; PAVITT, K. Nature and importance the national systems of innovation. Paris: OCDE, 1994. (STI Review, n.14).

PÊGO, B.; CAMPOS NETO, C.A. da S. (Orgs.). Biocombustíveis no Brasil: etanol e biodiesel. In: ____ ____. (Orgs.). Infraestrutura econômica no Brasil: diagnósticos e perspectivas para 2025. Brasília: Ipea, 2010. Livro 6, v.1, cap.4, p.193-250.

SUZIGAN, W.; ALBUQUERQUE, E. da M. A interação entre universidades e empresas em perspectiva histórica no Brasil. In: SUZIGAN, W. et al. (Orgs.). Em busca da inovação: interação universidade-empresa no Brasil. Belo Horizonte: Autêntica, 2011. p.17-43.

VIOTTI, E.B. Fundamentos e evolução dos indicadores de CT\&I. In: VIOTTI, E.B.; MACEDO, M. de M. (Orgs.). Indicadores de ciência, tecnologia e inovação. Campinas: UNICAMP, 2003. p.41-87.

National learning systems: a new approach on technological change in late industrializing economies and evidences from the cases of Brazil and South Korea. Technological Forecasting and Social Change, v.69, n.7, p.653-680, 2002. Disponível em: <http:// www.sciencedirect.com/science/article/pii/S0040162501001676> Acesso em: 15 mar. 2010. doi: 10.1016/S0040-1625(01)00167-6. 